\title{
Chronic Active Antibody-Mediated Rejection Is Associated With the Upregulation of Interstitial But Not Glomerular Transcripts
}

\author{
Andriy Trailin ${ }^{1}$, Petra Mrazova ${ }^{1}$, Petra Hruba ${ }^{1}$, Ludek Voska ${ }^{2}$, Eva Sticova ${ }^{2}$, \\ Antonij Slavcev $^{3}$, Marek Novotny ${ }^{4,5}$, Matej Kocik $^{6}$ and Ondrej Viklicky ${ }^{1,4^{*}}$ \\ 1 Transplant Laboratory, Institute for Clinical and Experimental Medicine, Prague, Czechia, ${ }^{2}$ Department of Clinical and \\ Transplant Pathology, Institute for Clinical and Experimental Medicine, Prague, Czechia, ${ }^{3}$ Department of Immunogenetics, \\ Institute for Clinical and Experimental Medicine, Prague, Czechia, ${ }^{4}$ Department of Nephrology, Transplant Centre, Institute for \\ Clinical and Experimental Medicine, Prague, Czechia, ${ }^{5}$ Institute of Physiology, $1^{\text {st }}$ Faculty of Medicine, Charles University, \\ Prague, Czechia, ${ }^{6}$ Transplantation Surgery Department, Institute for Clinical and Experimental Medicine, Prague, Czechia
}

\section{OPEN ACCESS}

Edited by: Geraldo Aleixo Passos, University of São Paulo, Brazil

Reviewed by:

Sistiana Aiello,

Istituto di Ricerche Farmacologiche

Mario Negri (IRCCS), Italy Lucy Sullivan,

The University of Melbourne, Australia

*Correspondence: Ondrej Viklicky onvi@ikem.cz

Specialty section:

This article was submitted to Alloimmunity and Transplantation,

a section of the journal

Frontiers in Immunology

Received: 23 June 2021 Accepted: 25 August 2021 Published: 20 September 2021

Citation:

Trailin A, Mrazova P, Hruba P, Voska L, Sticova E, Slavcev A, Novotny M, Kocik M and Viklicky O (2021) Chronic Active AntibodyMediated Rejection Is Associated With the Upregulation of Interstitial But Not Glomerular Transcripts.

Front. Immunol. 12:729558. doi: 10.3389/fimmu.2021.729558
Molecular assessment of renal allografts has already been suggested in antibody-mediated rejection (ABMR), but little is known about the gene transcript patterns in particular renal compartments. We used laser capture microdissection coupled with quantitative RT-PCR to distinguish the transcript patterns in the glomeruli and tubulointerstitium of kidney allografts in sensitized retransplant recipients at high risk of ABMR. The expressions of 13 genes were quantified in biopsies with acute active ABMR, chronic active ABMR, acute tubular necrosis (ATN), and normal findings. The transcripts were either compartment specific (TGFB1 in the glomeruli and HAVCR1 and IGHG1 in the tubulointerstitium), ABMR specific (GNLY), or follow-up specific (CXCL10 and CX3CR1). The transcriptional profiles of early acute ABMR shared similarities with ATN. The transcripts of CXCL10 and TGFB1 increased in the glomeruli in both acute ABMR and chronic active ABMR. Chronic active ABMR was associated with the upregulation of most genes (SH2D1B, CX3CR1, IGHG1, MS4A1, C5, CD46, and TGFB1) in the tubulointerstitium. In this study, we show distinct gene expression patterns in specific renal compartments reflecting cellular infiltration observed by conventional histology. In comparison with active ABMR, chronic active $A B M R$ is associated with increased transcripts of tubulointerstitial origin.

Keywords: kidney transplantation, antibody-mediated rejection, gene expression, laser capture microdissection, renal compartments

\section{INTRODUCTION}

Both acute antibody-mediated rejection (ABMR) and chronic ABMR are the main risk factors for late renal allograft loss (1-3). Sensitization to donor human leukocyte antigen (HLA) represents the main risk factor for ABMR development (4-6). Molecular diagnostics has already been suggested to improve the diagnostic accuracy of ABMR by histological assessment (7-9). Recent molecular 
methods evaluate biopsy specimens irrespective of the cortex or medulla origin due to the proportion of the cortex having little influence on the molecular diagnosis of rejection (10), while conventional histological assessment relies specifically on the cortex. The topography of intrarenal transcripts is important in understanding the mechanisms of ABMR; however, it has not yet been systemically studied, mainly due to technical obstacles.

In situ hybridization (ISH) is a powerful technique for the identification of specific messenger RNA (mRNA) expressions within individual cells in tissue sections, providing insights into physiological processes and disease pathogenesis. However, this complex method is technically difficult and requires the precise optimization of many steps for each tissue examined and for each probe used. Although many improvements have been made, the main limitation of ISH remains its poor sensitivity $(11,12)$. Aside from the NanoString platform (13), laser capture microdissection (LCM), when combined with molecular techniques, has demonstrated the ability to detect gene transcripts from distinct renal architecture (14). Until now, transcriptomic data based on LCM in renal allografts are limited (15-17) due to excessive necessary workload and the limited availability of sufficient tissue to enable highquality transcripts.

In this study, we used LCM to examine the compartmentspecific expression patterns of selected genes in sensitized kidney transplant recipients who suffered from active and chronic active ABMR.

\section{PATIENTS AND METHODS}

\section{Patient's Characteristics and Sample Collection}

A small portion $(\sim 2 \mathrm{~mm})$ of all for-cause and 3-month (3M) protocol biopsies, performed from January 2015 until January 2019 in sensitized kidney transplant recipients who had undergone a second, third, or a fourth kidney transplantation, were embedded into Tissue-Tek ${ }^{\circledR}$ O.C.T. Compound (Sakura Finetek USA, Inc., Torrance, CA, USA), snap frozen in liquid nitrogen immediately, and then stored at $-80^{\circ} \mathrm{C}$ for future LCM and gene expression analyses $(N=211)$. We identified patients with active ABMR (aABMR, $N=28$ ) and chronic active ABMR (caABMR, $N=36$ ). Biopsies with coincidental T-cell-mediated rejection (TCMR), borderline lesions, polyomavirus nephropathy, or recurrent disease were excluded. Biopsies from patients with acute tubular necrosis (ATN) diagnosed early after transplantation $(N=23)$ and normal findings at $3 \mathrm{M}$ protocol biopsies $(N=27)$ served as controls. Samples with absence of the renal cortex or glomeruli and samples with insufficient RNA quantity were excluded. The final cohort consisted of 43 patients (aABMR, $N=10$; caABMR, $N=10$; early ATN, $N=11$; and $3 \mathrm{M}$ normal findings, $N=12$ ) (Figure 1).

The demographics and clinical characteristics of the patients are summarized in Table 1. Both aABMR and caABMR were defined according to the recent Banff classification (7). All patients from the ATN group displayed ATN along with mild chronic lesions. All stable patients from the control group were negative for donor-specific antibodies (DSA) and exhibited only a mild degree of arteriosclerosis, arteriolar hyalinosis, and interstitial fibrosis and tubular atrophy (IFTA) on biopsy. Detailed Banff scores of all the studied groups are given in Supplementary Table S1.

The present research was carried out in accordance with ethical standards and was approved by the Ethics Committee of the Institute for Clinical and Experimental Medicine in Prague (no. A 13-02-01). All patients gave written informed consent for participation in the study.

\section{Laser Capture Microdissection}

All biopsies were performed using a semiautomatic biopsy gun with 16-G needle (Möller Medical GmbH, Fulda, Germany) guided by ultrasound. A 2-mm piece of biopsy tissue was embedded into Tissue-Tek, snap frozen with liquid nitrogen, and then kept at $-80^{\circ} \mathrm{C}$ for future LCM.

Sections $10 \mu \mathrm{m}$ thick were cut on a cryomicrotome (Leica CM1950, Wetzlar, Germany) at $-20^{\circ} \mathrm{C}$. Up to five sections were mounted onto each RNase-free MMI Membrane Slide (MMI AG, Glattbrugg, Switzerland). The slides were stored inside the cryochamber until the last section was cut and then, without defrosting, the sections were immediately fixed in ice-cold $96 \%$ ethanol for 45 s. After removal of Tissue-Tek in ice-cold 70\% ethanol, the sections were rehydrated in ice-cold RNase-free water, stained in hematoxylin for $30 \mathrm{~s}$, rinsed in RNase-free water, stained by eosin for $45 \mathrm{~s}$ (H\&E staining kit for LCM; MMI AG, Glattbrugg, Switzerland), rinsed again in RNase-free water, and then dehydrated in two portions of ice-cold $100 \%$ ethanol for 45 and $60 \mathrm{~s}$. The slides were then air dried, inverted, and placed onto a glass object slide for protection against contamination. Then, the sections were immediately microdissected using the inverted Olympus IX70 microscope (Olympus, Hamburg, Germany) coupled with the microdissection system MMI CellCut ${ }^{\circledR}$ (Molecular Machines\&Industries, Zürich, Switzerland), which uses low-damage UV laser.

All available glomeruli (except those with global glomerulosclerosis) were delineated excluding Bowman's capsule. Tubulointerstitial areas, which included the cortical labyrinth, medullary rays, and the surrounding interstitium with peritubular capillaries, were outlined as well. Selected compartments were dissected by a laser microbeam through the objective and collected onto the adhesive lid of $0.5 \mathrm{ml} \mathrm{MMI}$ isolation caps (MMI AG, Glattbrugg, Switzerland). For each biopsy, a median of 61 glomerular cross-sections ( $\min -\max =$ 11-173), corresponding to a median area of $1.36 \mathrm{~mm}^{2}$ (min$\max =0.34-4.00 \mathrm{~mm}^{2}$ ), and the cortical tubulointerstitium (TI) with a median area of $7.50 \mathrm{~mm}^{2}\left(\min -\max =1.93-17.10 \mathrm{~mm}^{2}\right)$ were collected. All procedures were performed under strict RNase-free conditions. The microdissection time did not exceed $30 \mathrm{~min}$ to avoid the action of RNases (as was established in preliminary experiments).

\section{RNA Extraction, cDNA Synthesis, and Real-Time PCR}

The microdissected tissue samples were immediately homogenized by vortexing in RLT lysis buffer on the basis of 


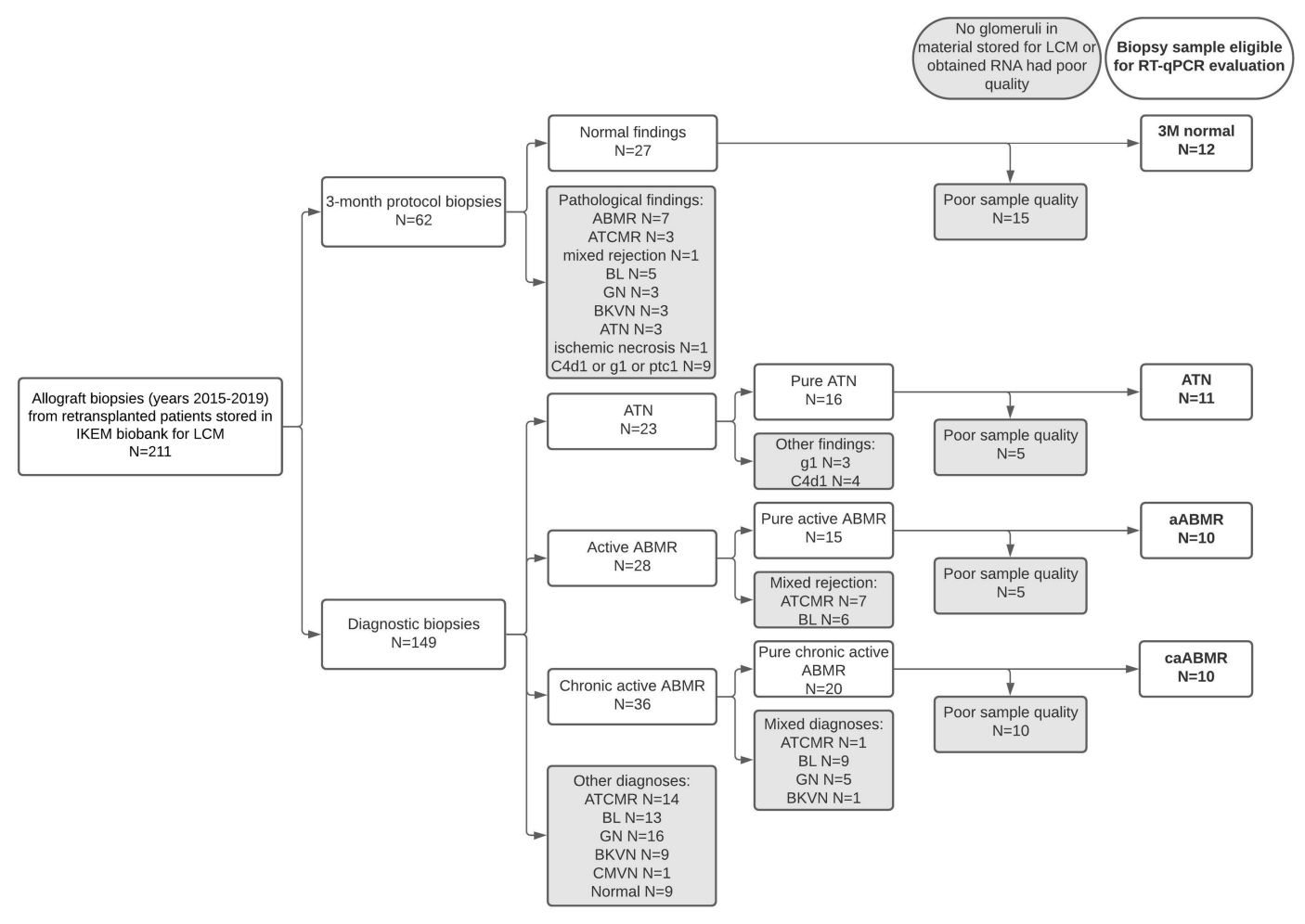

FIGURE 1 | Flowchart of samples selection. Only samples with pure diagnosis of active ABMR, chronic active ABMR, ATN and normal findings in 3-month protocol biopsy, which had enough material for LCM and good RNA quality, were used for RT-qPCR form the biobank of biopsies from retransplanted patients. LCM, laser capture microdissection; ABMR, antibody-mediated rejection; ATCMR, active T-cell mediated rejection; BL, borderline lesions; GN, recurrence of glomerulonephritis; BKVN, BK virus nephropathy; CMVN, cytomegalovirus nephropathy; ATN, acute tubular necrosis.

guanidine thiocyanate (RNeasy Micro Kit, Qiagen, Hilden, Germany), containing $1 \%$ of $\beta$-mercaptoethanol (SigmaAldrich, Taufkirchen, Germany), and stored at $-20^{\circ} \mathrm{C}$ until RNA isolation. For each patient, all dissected glomeruli or TI were pooled for RNA extraction. Total RNA was isolated using the RNeasy Micro Kit (Qiagen, Hilden, Germany) according to the manufacturer's instructions. Reverse transcription was performed using SuperScript II reverse transcriptase (Invitrogen, Thermo Fisher Scientific, Waltham, MA, USA). Only the samples with $\mathrm{Ct}_{\text {GAPDH }}$ values $<32$ were included.

Due to the limited amount of material obtained by LCM, which was not suitable for whole-transcriptome analysis, gene expression profiling using a custom-made TaqMan low-density array (TLDA) cards (Applied Biosystems, Thermo Fisher Scientific, Waltham, MA, USA) enabling the measurement of 16 genes for eight samples per one card was used. Fourteen genes of interest were selected for expression profiling based on literature data regarding their implications in cellular immunity, humoral immunity, and injury/inflammatory response, plus glyceradyhyde3-phosphate dehydrogenase $(G A P D H)$ as the endogenous control and Nephrosis 2, Idiopathic, Steroid-Resistant, Podocin (NPHS2) as the gene expressed mainly in the glomeruli (Supplementary Table S2). All genes were measured in triplicate. Real-time quantitative reverse transcription PCR (RT-qPCR) amplification was performed on an ABI Prism ${ }^{\circledR} 7900$ H.T. Sequence Detection system (Applied Biosystems, Thermo Fisher Scientific, Waltham, MA, USA) using TaqMan Fast Advanced master mix (Applied Biosystems, Thermo Fisher Scientific, Waltham, MA, USA). The $2^{-\Delta \Lambda \mathrm{Ct}}$ method with GAPDH as an internal standard was used for quantification of the target gene expression. As a calibrator, one sample of TI from samples with normal histological findings at the 3-month protocol biopsy with good expression profiles of all the target genes was chosen. Gene expression data were normalized to GAPDH and relative to the calibrator sample (RQ manager 1.2. software for automated data analysis; Applied Biosystems, Thermo Fisher Scientific, Waltham, MA, USA), $\log 2$ transformed, and presented as fold change (FC) values. As the TNFRSF17 gene was detectable in less than $15 \%$ of samples, it was excluded from all further analyses. As a control of sampling accuracy, glomerulusspecific NPHS2 (podocin) was used (Supplementary Figure S1).

\section{Statistical Analysis}

Differences in the gene expression or clinical parameters between groups were analyzed using the Mann-Whitney test or the chisquare test, where appropriate. Spearman's rank correlation tests were performed for correlation analyses. Statistical analyses were performed using SPSS v.20.0 (SPSS, Inc., Chicago, IL, USA) and GraphPad Prism 5 v.5.03 for Windows (GraphPad Software, San Diego, CA, USA). Statistical significance was set at $p<0.05$. Data are presented as median (minimum-maximum). 
TABLE 1 | Patients' demographics and clinical characteristics according to the studied groups.

\begin{tabular}{|c|c|c|c|c|c|c|}
\hline & ATN & Active ABMR & $p$-value & Normal & Chronic active ABMR & $p$-value \\
\hline$N$ & 11 & 10 & & 12 & 10 & \\
\hline Biopsy follow-up (POD) ${ }^{a}$ & $9(6-48)$ & $10(5-144)$ & 0.456 & $100(84-110)$ & $2,004(231-4,607)$ & $<0.001$ \\
\hline Serum creatinine at biopsy $(\mu \mathrm{mol} / \mathrm{L})^{\mathrm{a}}$ & $299.7(137.0-754.5)$ & $294.2(65.8-806.4)$ & 0.439 & $121.6(62.2-134.6)$ & $179.4(142.8-376.7)$ & $<0.001$ \\
\hline Age $\left(\right.$ years) ${ }^{a}$ & $48(39-74)$ & $39(26-64)$ & 0.078 & $53(35-68)$ & $46(27-60)$ & 0.080 \\
\hline Male, N (\%) & $9(82)$ & $8(80)$ & 1.000 & $8(67)$ & $9(90)$ & 0.323 \\
\hline No. of transplants (second/third/fourth) & $9 / 2 / 0$ & $7 / 2 / 1$ & 0.547 & $10 / 2 / 0$ & $6 / 3 / 1$ & 0.361 \\
\hline Dialysis vintage (months) ${ }^{a, b}$ & $58(37-238)$ & $58(26-154)$ & 0.778 & $55(11-103)$ & $58(13-232)$ & 0.872 \\
\hline PRA $\max , N(\%)^{a}$ & $10(0-90)$ & $75(20-98)$ & 0.022 & $19(2-96)$ & $80(2-98)$ & 0.050 \\
\hline Anti-HLA class I and/or II positive, $N(\%)^{\mathrm{C}}$ & $10(91)$ & $10(100)$ & 1.000 & $8(44)$ & $10(100)$ & 0.096 \\
\hline DSA class I and/or II positive, $N(\%)^{\mathrm{C}}$ & $6(55)$ & $9(90)$ & 0.149 & $0(0)$ & $5(50)$ & 0.01 \\
\hline Anti-MICA positive, $N(\%)^{\mathrm{C}}$ & $0(0)$ & $3(30)$ & 0.090 & $1(8.3)$ & $3(33.3)$ & 0.272 \\
\hline Induction, $N$ & & & 0.543 & & & 0.244 \\
\hline None & 0 & 0 & & 0 & 1 & \\
\hline Basiliximab/daclizumab & 1 & 0 & & 0 & 1 & \\
\hline ATG & 7 & 8 & & 12 & 7 & \\
\hline ATG, rituximab & 3 & 2 & & 0 & 1 & \\
\hline Donors' age (years) ${ }^{\mathrm{a}}$ & 63 (39-73) & $50(35-58)$ & 0.024 & $55(36-65)$ & $52(7-65)$ & 0.372 \\
\hline Donor type (D), $N(\%)$ & $11(100)$ & $10(100)$ & 1.000 & $11(92)$ & $9(90)$ & 1.000 \\
\hline Male, $N(\%)$ & $5(46)$ & $6(60)$ & 0.670 & $8(67)$ & $7(70)$ & 1.000 \\
\hline ECD, $N(\%)$ & $6(55)$ & $3(33)$ & 0.387 & 4 (33.3) & $6(66.7)$ & 0.198 \\
\hline CIT $(h)^{a}$ & $14.3(9.1-23.2)$ & $16.2(10.3-20.6)$ & 0.573 & $14.9(3.4-20.5)$ & $18.4(0-23)$ & 0.118 \\
\hline Mismatches $^{a}$ & $2(0-6)$ & $4(2-6)$ & 0.029 & $3(0-5)$ & $4.5(2-6)$ & 0.008 \\
\hline
\end{tabular}

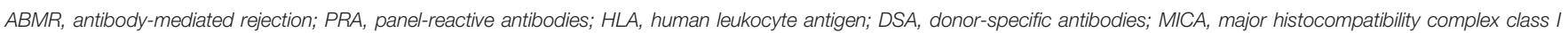
chain-related antigen A; ATG, anti-thymocyte globulin; D, deceased; ECD, expanded criteria donor; CIT, cold ischemia time.

${ }^{a}$ Data are presented as median (minimum-maximum).

${ }^{b}$ Total time on dialysis.

${ }^{c}$ At the time of biopsy.

\section{RESULTS}

\section{Gene Expression Upregulation in ABMR}

In this study, we evaluated transcripts thought to be associated with alloimmune response during ABMR (Supplementary Table S2). Initially, we compared the transcript expression patterns in the glomeruli and TI during ABMR and in controls, either samples from ATN as comparator for acute active ABMR or samples from normal findings as comparator for chronic active ABMR (Supplementary Figure S2).

The glomeruli in aABMR showed upregulation of the chemokine CXCL10 compared to ATN, while the TI in aABMR exhibited increased IFNG signals (Supplementary Figure S2A). In caABMR, the intrarenal gene expressions of GNLY, CX3CR1, TGFB1, and CXCL10 were upregulated in both the glomeruli and TI compared to normal findings at 3 months (Supplementary Figure S2A). The glomeruli of caABMR biopsies showed the upregulation of CDI4 and IFNG compared to normal control samples, whereas higher expressions of $S H 2 D 1 B$ and IRF4 were observed in the TI (Supplementary Figure S2B).

\section{Differences in Early Active ABMR and Chronic Active ABMR}

Chronic active ABMR differed from early active ABMR mainly in TI-associated transcript levels (Figure 2). Only one transcript, CD14, showed significant upregulation in the glomerular compartment of caABMR samples compared with aABMR $(p=0.027)$. The expressions of CX3CR1, SH2D1B, IGHG1,
IRF4, and MS4A1, the complement-associated transcripts C5 and CD46, and the TGFB1 transcript were significantly upregulated in the TI in chronic active ABMR compared to active ABMR samples (Figure 2).

Therefore, genes associated with immune response were markedly upregulated in chronic ABMR compared to acute ABMR, especially in the interstitium, which suggests enhanced inflammation and fibrogenesis. This finding also corresponds well to conventional histology describing a higher total inflammation score. There was a strong correlation between the expressions of CX3CR1, IFNG, IRF4, and TGFB1 in the TI and the Banff total inflammation score ( $p<0.001$; Supplementary Table S3).

\section{Different Gene Expression Patterns in Renal Compartments}

Finally, we compared the gene expression patterns between the glomeruli and TI in all groups (Supplementary Figure S3 and Figure 3). Regardless of the diagnosis, the TGFB1 transcript was more highly expressed in the glomeruli while IGHG1 and HAVCR1 in the TI in all samples.

Non-rejection samples from ATN and normal findings exhibited higher glomerular expressions of GNLY, CXCL10, CX3CR1, and CD46, while there were higher TI expressions of IGHG1, MS4A1, IRF4, and C5 (Supplementary Figure S2). In active ABMR samples, a higher glomerular expression of CX3CR1 was described, while the upregulation of IGHG1 was observed in the TI. In chronic active ABMR, CXCL10 was more highly expressed in the glomeruli, while MS4A1 and IRF4 showed higher expressions in the TI (Figure 3). 

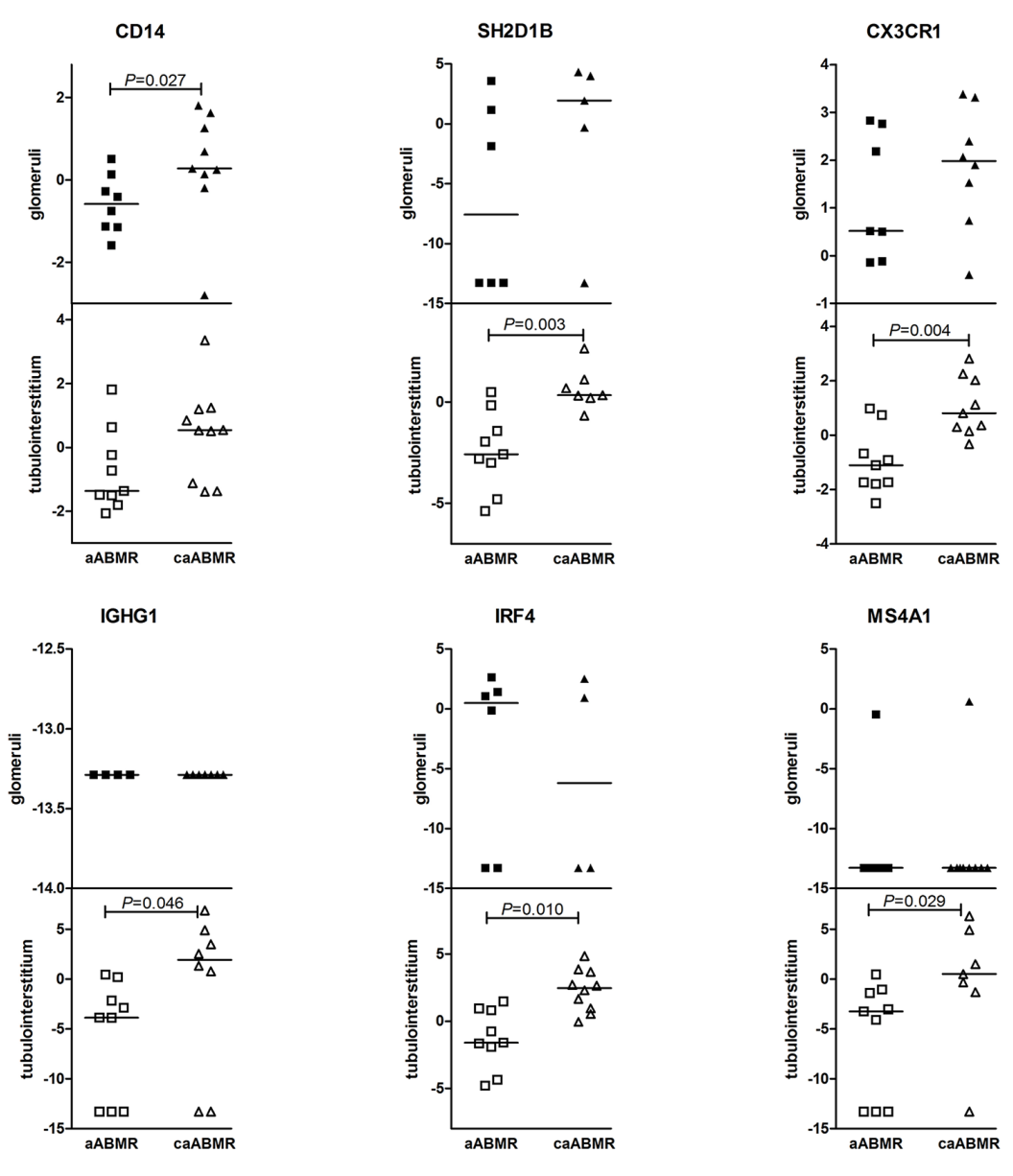

TGFB1

C5
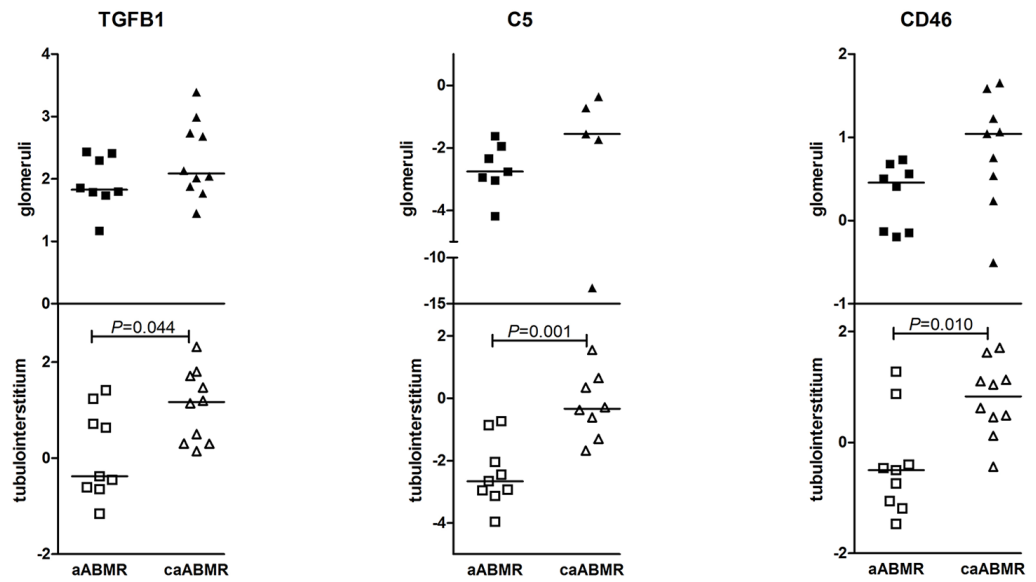

FIGURE 2 | Differences in gene expression (log2 FC) between active ABMR (aABMR) and chronic active ABMR (caABMR) in glomeruli (dark marks) and tubulointerstitium (empty marks). Differences are calculated by Mann-Whitney test.

\section{DISCUSSION}

The expressions of many genes of native kidney are compartment specific $(18,19)$. The gene expression patterns in inflamed kidneys during various kidney diseases or allograft rejection are supposed to correspond to the cellular infiltration observed in conventional histology. Despite current achievements in molecular diagnostics in kidney transplantation, little is known about renal compartment gene expression patterns in transplant pathologies. In this study, we used LCM to distinguish the gene expression 


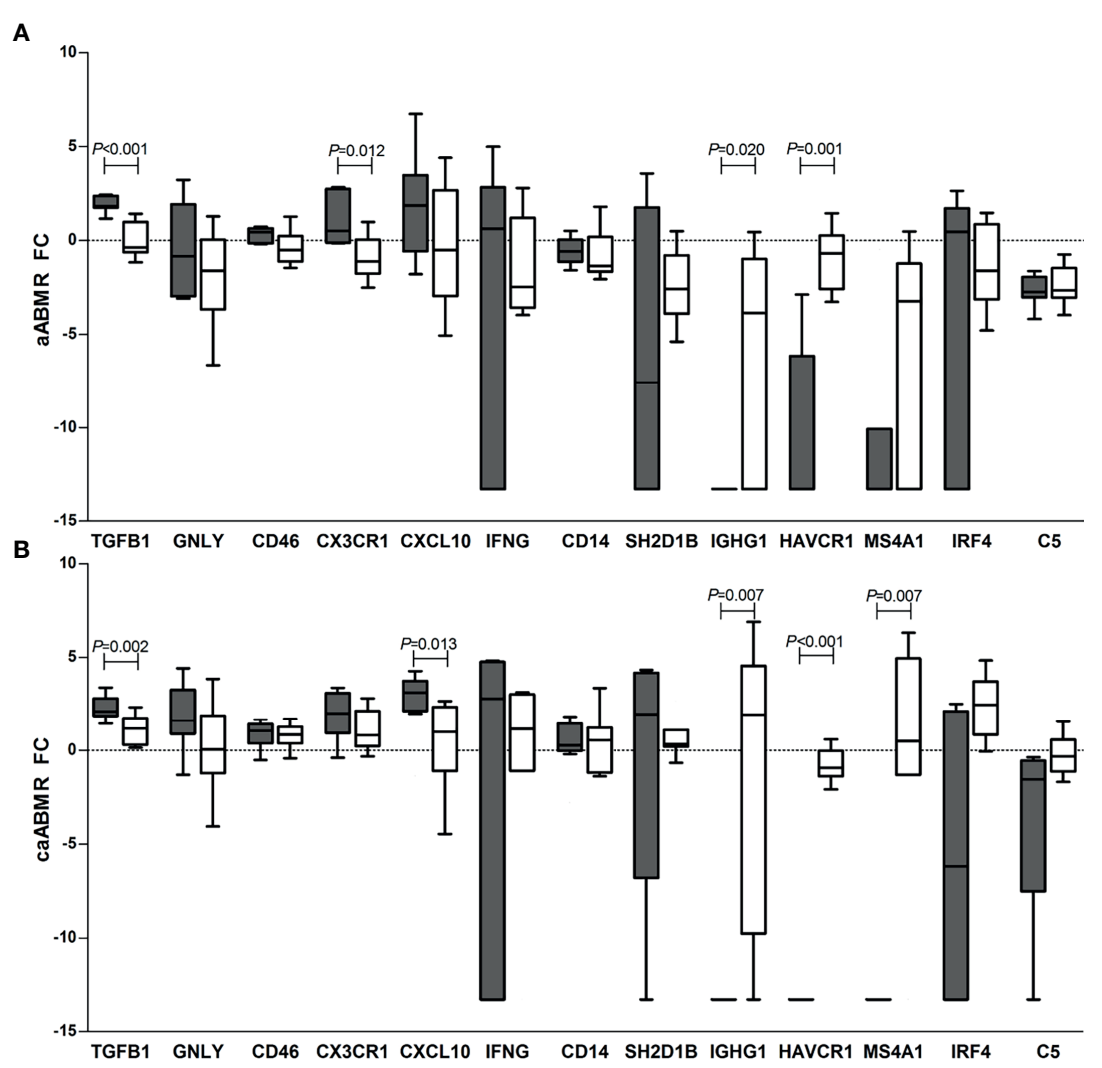

FIGURE 3 | Differences in gene expression (log2 FC) between glomeruli (G, dark bars) and tubulointerstitium (TI, empty bars) in the groups with: (A) active ABMR (aABMR, $n=10$ ), and $(\mathbf{B})$ chronic active ABMR (caABMR, $n=10$ ). Boxes extends from the 25th to 75 th percentiles, lines in the middle of the box shows median and whiskers indicate the highest and lowest value within inner fences (Tukey whiskers). Differences are calculated by Mann-Whitney test.

patterns in the glomeruli and the tubulointerstitial area of kidney allografts in sensitized kidney retransplant recipients who were at high risk of ABMR development.

We found different expressions of transcripts associated with innate and adaptive immune responses in the glomerular and tubulointerstitial compartments. While the expressions of some genes associated with cellular infiltrates were increased in the glomeruli, others predominated in the TI. Contradictory data on gene expression patterns in various kidney graft compartments have been reported so far, mainly due to less advanced techniques and various diagnoses in stable grafts and during rejection. Of note is that strong correlations were reported between chromogenic ISH signals for chemokine transcripts and gene expression levels using the NanoString platform (13). Compared with the NanoString technology, RNA ISH and LCM coupled with RT-qPCR allow precisely assessing sites of gene expression. Previously, several studies have applied LCM coupled with RT-qPCR to evaluate the compartment-specific patterns of gene expression for $\operatorname{ACE}(20,21)$, ion channels $(22)$, cytokines $(23,24)$, and CD molecules $(25,26)$. The NanoString ${ }^{\circledR}$ nCounter ${ }^{\circledR}$ gene expression system has been recently shown to evaluate archival formalin-fixed paraffinembedded tissues $(27,28)$. This technology allows gene expression analysis to be performed on the same tissue assessed with histology, permitting direct molecular-histologic correlations (29). Although this technology enables measuring up to 800 selected probes, its classification accuracy for kidney graft rejection based on formalinfixed samples has not been validated in larger cohorts yet.

The antibody-endothelial interaction is associated with cellular infiltration of the glomeruli and peritubular capillaries. The Banff criteria for ABMR include microvascular inflammation (MVI) characterized by glomerulitis (g) and/or peritubular capillarities (ptc). Glomerular infiltration with monocyte/macrophages or natural killer $(\mathrm{NK})$ cells has been frequently reported in ABMR (30-33), while the tubulointerstitial area was reported to be infiltrated to a less extent (30). However, in chronic active ABMR, the glomeruli displayed fewer T- and B-cell infiltrates than did the TI (34). B-cell aggregates in the TI were frequently observed as well, which is consistent with our findings describing the B-cell-related transcripts IGHG1 and MS4A1 to be upregulated in the TI in chronic active ABMR.

In our study, the transcriptional profile of active ABMR was found to be similar to ATN. Clearly, 8 of the 11 patients with ATN had DSA at transplantation. Therefore, it is likely that some of the ATN cases had subclinical ABMR, which could not be detected by routine histological assessment. Of note is that 4 of the 10 active ABMR patients exhibited ATN-like patterns.

The gene expressions in chronic active ABMR were higher than in those with early acute ABMR; the differences were 
obvious especially in the TI, as most of the patients exhibited higher Banff total inflammation scores. The expressions of the SH2D1B, CX3CR1, IGHG1, IRF4, MS4A1, C5, CD46, and TGFB1 transcripts were higher in the TI of patients with chronic active ABMR; only the expression of the CD14 gene predominated in the glomeruli. The upregulation of complement-associated gene transcripts in chronic ABMR was just recently reported (35). In our study, we showed the TI as a source of upregulated transcripts, including complement ones, in chronic active ABMR. The predominant sites of complement synthesis are renal tubules, but inflammatory cells can also contribute to complement production in inflamed tissues (36). The lower tubulointerstitial expression of C5 from the samples obtained early after transplantation (ATN, active ABMR) may reflect tubular damage associated with early ischemic injury.

Interestingly, Cohen et al. (37) used LCM coupled with RTqPCR in a small study and showed the upregulation of CXCL10 expression in each renal compartment during severe rejection. In our study, the increased CXCL10 expressions in both chronic active $A B M R$ and active $A B M R$ were observed mainly in the glomeruli. The CXCL10 (IP10) chemokine was shown to be less specific, and its higher gene or protein expression reflects renal injury regardless of the origin. Similarly, TGFB1 mRNA expression was shown to be expressed in both scarred or inflamed glomeruli and interstitium (38-40). Previously, we have shown that grafts with lower expressions of several transcripts, such as TGFB1 or chemokines, were associated with poor kidney allograft outcomes (41). Similarly, in the present study, we observed the lowest TGFB1 expression in grafts with active ABMR diagnosed early after transplantation. Nevertheless, our study was not powered to study the associations of gene transcripts with graft outcomes. Our observation of the upregulation of the monocyte marker CD14 in the glomeruli of patients with chronic active ABMR is in line with previous studies that showed recruitment of monocytes into the glomerular compartment in ABMR (42). The observed compartment transcriptomic specificity reflects distinct cellular infiltrates that dominate either in the glomeruli or in the interstitium $(26,43)$.

The limitation of our study was the analysis of only several predefined transcripts due to the low RNA yield from laserdissected glomeruli and TI, which does not allow either microarray RNA evaluation or RNA sequencing. Relative quantification of gene expression also limits the pathophysiological conclusions. Nevertheless, our study is the first systematically evaluating RNA transcripts from renal compartments in defined transplant pathology, while previous studies have mainly used immunohistochemistry for protein analyses or have focused on native kidneys. Clearly, the patient numbers are low to study outcomes, however high enough to see the differences in renal compartments. Of note is that the LCM method seems not suitable for routine assessments due to its extreme time requirements for sample preparation.

\section{REFERENCES}

1. Sellares J, de Freitas DG, Mengel M, Reeve J, Einecke G, Sis B, et al. Understanding the Causes of Kidney Transplant Failure: The Dominant
In conclusion, in this study, we showed distinct gene expression patterns in specific renal compartments reflecting cellular infiltration observed by conventional histology. In comparison with active $A B M R$, chronic active ABMR is associated with the increased transcripts of tubulointerstitial origin.

\section{DATA AVAILABILITY STATEMENT}

The original contributions presented in the study are included in the article/Supplementary Material. Further inquiries can be directed to the corresponding author.

\section{ETHICS STATEMENT}

The studies involving human participants were reviewed and approved by the Ethics Committee of the Institute for Clinical and Experimental Medicine in Prague (No. A 13-02-01). The patients/participants provided their written informed consent to participate in this study.

\section{AUTHOR CONTRIBUTIONS}

$\mathrm{AT}$ and PM performed the research and wrote the manuscript. $\mathrm{PH}$ performed the research and statistical analysis. LV, ES, AS, $\mathrm{MN}$, and MK performed the research. OV supervised the project and wrote the manuscript. All authors contributed to the article and approved the submitted version.

\section{FUNDING}

This study was supported by the EU under ESIF, Operational Programme Research, Development and Education, CAMERA Project (CZ.02.2.69/0.0/0.0/17_050/0008096), and by the Ministry of Health of the Czech Republic under grants NV1906-00031 and NU21-06-00021 and its conceptual development of research organizations (Institute for Clinical and Experimental Medicine-IKEM, IN 00023001).

\section{SUPPLEMENTARY MATERIAL}

The Supplementary Material for this article can be found online at: https://www.frontiersin.org/articles/10.3389/fimmu.2021. 729558/full\#supplementary-material

Role of Antibody-Mediated Rejection and Nonadherence. Am J Transplant (2012) 12:388-99. doi: 10.1111/j.1600-6143.2011.03840.x

2. Halloran PF, Chang J, Famulski K, Hidalgo LG, Salazar ID, Merino Lopez M, et al. Disappearance of $\mathrm{T}$ Cell-Mediated Rejection Despite Continued 
Antibody-Mediated Rejection in Late Kidney Transplant Recipients. J Am Soc Nephrol (2015) 26:1711-20. doi: 10.1681/ASN.2014060588

3. Gaston RS, Cecka JM, Kasiske BL, Fieberg AM, Leduc R, Cosio FC, et al. Evidence for Antibody-Mediated Injury as a Major Determinant of Late Kidney Allograft Failure. Transplantation (2010) 90:68-74. doi: 10.1097/ TP.0b013e3181e065de

4. Eikmans M, Waanders MM, Roelen DL, van Miert PP, Anholts JD, de Fijter HW, et al. Differential Effect of Pretransplant Blood Transfusions on Immune Effector and Regulatory Compartments in HLA-Sensitized and Nonsensitized Recipients. Transplantation (2010) 90:1192-9. doi: 10.1097/TP.0b013e31 81fa943d

5. Terasaki PI, Ozawa M. Predictive Value of HLA Antibodies and Serum Creatinine in Chronic Rejection: Results of a 2-Year Prospective Trial. Transplantation (2005) 80:1194-7. doi: 10.1097/01.tp.0000174338. 97313.5a

6. Aubert O, Loupy A, Hidalgo L, Duong van Huyen JP, Higgins S, Viglietti D, et al. Antibody-Mediated Rejection Due to Preexisting Versus De Novo Donor-Specific Antibodies in Kidney Allograft Recipients. J Am Soc Nephrol (2017) 28:1912-23. doi: 10.1681/ASN.2016070797

7. Loupy A, Haas M, Roufosse C, Naesens M, Adam B, Afrouzian M, et al. The Banff 2019 Kidney Meeting Report (I): Updates on and Clarification of Criteria for T Cell- and Antibody-Mediated Rejection. Am J Transplant (2020) 20:2318-31. doi: 10.1111/ajt.15898

8. Haas M, Loupy A, Lefaucheur C, Roufosse C, Glotz D, Seron D, et al. The Banff 2017 Kidney Meeting Report: Revised Diagnostic Criteria for Chronic Active T Cell-Mediated Rejection, Antibody-Mediated Rejection, and Prospects for Integrative Endpoints for Next-Generation Clinical Trials. Am J Transplant (2018) 18:293-307. doi: 10.1111/ajt.14625

9. Halloran PF, Reeve J, Akalin E, Aubert O, Bohmig GA, Brennan D, et al. Real Time Central Assessment of Kidney Transplant Indication Biopsies by Microarrays: The INTERCOMEX Study. Am J Transplant (2017) 17:285162. doi: 10.1111/ajt.14329

10. Madill-Thomsen KS, Wiggins RC, Eskandary F, Bohmig GA, Halloran PF. The Effect of Cortex/Medulla Proportions on Molecular Diagnoses in Kidney Transplant Biopsies: Rejection and Injury Can Be Assessed in Medulla. Am J Transplant (2017) 17:2117-28. doi: 10.1111/ajt.14233

11. Mahmood R, Mason I. In-Situ Hybridization of Radioactive Riboprobes to RNA in Tissue Sections. Methods Mol Biol (2008) 461:675-86. doi: 10.1007/ 978-1-60327-483-8_45

12. Speel EJ, Hopman AH, Komminoth P. Amplification Methods to Increase the Sensitivity of In Situ Hybridization: Play Card(s). J Histochem Cytochem (1999) 47:281-8. doi: 10.1177/002215549904700302

13. Sigdel T, Nguyen M, Liberto J, Dobi D, Junger H, Vincenti F, et al. Assessment of 19 Genes and Validation of CRM Gene Panel for Quantitative Transcriptional Analysis of Molecular Rejection and Inflammation in Archival Kidney Transplant Biopsies. Front Med (Lausanne) (2019) 6:213. doi: $10.3389 /$ fmed.2019.00213

14. Kohda Y, Murakami H, Moe OW, Star RA. Analysis of Segmental Renal Gene Expression by Laser Capture Microdissection. Kidney Int (2000) 57:321-31. doi: 10.1046/j.1523-1755.2000.00824.x

15. Rodder S, Scherer A, Korner M, Eisenberger U, Hertig A, Raulf F, et al. MetaAnalyses Qualify Metzincins and Related Genes as Acute Rejection Markers in Renal Transplant Patients. Am J Transplant (2010) 10:286-97. doi: 10.1111/ j.1600-6143.2009.02928.x

16. Serinsoz E, Bock O, Gwinner W, Schwarz A, Haller H, Kreipe H, et al. Local Complement C3 Expression Is Upregulated in Humoral and Cellular Rejection of Renal Allografts. Am J Transplant (2005) 5:1490-4. doi: 10.1111/j.1600-6143.2005.00873.x

17. Agustian PA, Bockmeyer CL, Modde F, Wittig J, Heinemann FM, Brundiers S, et al. Glomerular mRNA Expression of Prothrombotic and Antithrombotic Factors in Renal Transplants With Thrombotic Microangiopathy. Transplantation (2013) 95:1242-8. doi: 10.1097/TP.0b013e318291a298

18. Disset A, Cheval L, Soutourina O, Duong Van Huyen JP, Li G, Genin C, et al. Tissue Compartment Analysis for Biomarker Discovery by Gene Expression Profiling. PloS One (2009) 4:e7779. doi: 10.1371/journal.pone. 0007779

19. Chabardes-Garonne D, Mejean A, Aude JC, Cheval L, Di Stefano A, Gaillard MC, et al. A Panoramic View of Gene Expression in the Human
Kidney. Proc Natl Acad Sci USA (2003) 100:13710-5. doi: 10.1073/ pnas. 2234604100

20. Reich HN, Oudit GY, Penninger JM, Scholey JW, Herzenberg AM. Decreased Glomerular and Tubular Expression of ACE2 in Patients With Type 2 Diabetes and Kidney Disease. Kidney Int (2008) 74:1610-6. doi: 10.1038/ ki.2008.497

21. Wang G, Lai FM, Lai KB, Chow KM, Kwan CH, Li KT, et al. Discrepancy Between Intrarenal Messenger RNA and Protein Expression of ACE and ACE2 in Human Diabetic Nephropathy. Am J Nephrol (2009) 29:524-31. doi: $10.1159 / 000185629$

22. Ceol M, Tiralongo E, Baelde HJ, Vianello D, Betto G, Marangelli A, et al. Involvement of the Tubular ClC-Type Exchanger ClC-5 in Glomeruli of Human Proteinuric Nephropathies. PloS One (2012) 7:e45605. doi: 10.1371/ journal.pone. 0045605

23. Wang Y, Ito S, Chino Y, Goto D, Matsumoto I, Murata H, et al. Laser Microdissection-Based Analysis of Cytokine Balance in the Kidneys of Patients With Lupus Nephritis. Clin Exp Immunol (2010) 159:1-10. doi: 10.1111/j.1365-2249.2009.04031.x

24. Chan RW, Lai FM, Li EK, Tam LS, Chow KM, Lai KB, et al. Intrarenal Cytokine Gene Expression in Lupus Nephritis. Ann Rheum Dis (2007) 66:886-92. doi: 10.1136/ard.2006.063123

25. Heller F, Lindenmeyer MT, Cohen CD, Brandt U, Draganovici D, Fischereder $\mathrm{M}$, et al. The Contribution of B Cells to Renal Interstitial Inflammation. Am J Pathol (2007) 170:457-68. doi: 10.2353/ajpath.2007.060554

26. Segerer S, Heller F, Lindenmeyer MT, Schmid H, Cohen CD, Draganovici D, et al. Compartment Specific Expression of Dendritic Cell Markers in Human Glomerulonephritis. Kidney Int (2008) 74:37-46. doi: 10.1038/ ki.2008.99

27. Adam B, Afzali B, Dominy KM, Chapman E, Gill R, Hidalgo LG, et al. Multiplexed Color-Coded Probe-Based Gene Expression Assessment for Clinical Molecular Diagnostics in Formalin-Fixed Paraffin-Embedded Human Renal Allograft Tissue. Clin Transplant (2016) 30:295-305. doi: $10.1111 /$ ctr.12689

28. Dominy KM, Willicombe M, Al Johani T, Beckwith H, Goodall D, Brookes P, et al. Molecular Assessment of C4d-Positive Renal Transplant Biopsies Without Evidence of Rejection. Kidney Int Rep (2019) 4:148-58. doi: 10.1016/j.ekir.2018.09.005

29. Adam BA, Kikic Z, Wagner S, Bouatou Y, Gueguen J, Drieux F, et al. Intragraft Gene Expression in Native Kidney BK Virus Nephropathy Versus T CellMediated Rejection: Prospects for Molecular Diagnosis and Risk Prediction. Am J Transplant (2020) 20:3489-501. doi: 10.1111/ajt.15980

30. Magil AB, Tinckam K. Monocytes and Peritubular Capillary C4d Deposition in Acute Renal Allograft Rejection. Kidney Int (2003) 63:1888-93. doi: $10.1046 / j .1523-1755.2003 .00921 . x$

31. Halloran PF, Wadgymar A, Ritchie S, Falk J, Solez K, Srinivasa NS. The Significance of the Anti-Class I Antibody Response. I. Clinical and Pathologic Features of Anti-Class I-Mediated Rejection. Transplantation (1990) 49:8591. doi: 10.1097/00007890-199001000-00019

32. Venner JM, Hidalgo LG, Famulski KS, Chang J, Halloran PF. The Molecular Landscape of Antibody-Mediated Kidney Transplant Rejection: Evidence for NK Involvement Through CD16a Fc Receptors. Am J Transplant (2015) 15:1336-48. doi: 10.1111/ajt.13115

33. Yazdani S, Callemeyn J, Gazut S, Lerut E, de Loor H, Wevers M, et al. Natural Killer Cell Infiltration Is Discriminative for Antibody-Mediated Rejection and Predicts Outcome After Kidney Transplantation. Kidney Int (2019) 95:18898. doi: 10.1016/j.kint.2018.08.027

34. Sablik KA, Jordanova ES, Pocorni N, Clahsen-van Groningen MC, Betjes MGH. Immune Cell Infiltrate in Chronic-Active Antibody-Mediated Rejection. Front Immunol (2019) 10:3106. doi: 10.3389/fimmu.2019.03106

35. Cernoch M, Hruba P, Kollar M, Mrazova P, Stranavova L, Lodererova A, et al. Intrarenal Complement System Transcripts in Chronic Antibody-Mediated Rejection and Recurrent IgA Nephropathy in Kidney Transplantation. Front Immunol (2018) 9:2310. doi: 10.3389/fimmu.2018.02310

36. Sacks SH, Zhou W, Sheerin NS. Complement Synthesis in the Injured Kidney: Does It Have a Role in Immune Complex Glomerulonephritis? J Am Soc Nephrol (1996) 7:2314-9. doi: 10.1681/ASN.V7112314

37. Cohen CD, Grone HJ, Grone EF, Nelson PJ, Schlondorff D, Kretzler M. Laser Microdissection and Gene Expression Analysis on Formaldehyde-Fixed 
Archival Tissue. Kidney Int (2002) 61:125-32. doi: 10.1046/j.15231755.2002.00112.x

38. Horvath LZ, Friess H, Schilling M, Borisch B, Deflorin J, Gold LI, et al. Altered Expression of Transforming Growth Factor- $\beta$ s in Chronic Renal Rejection. Kidney Int (1996) 50:489-98. doi: 10.1038/ki.1996.340

39. Kaminska D, Tyran B, Mazanowska O, Letachowicz W, Kochman A, Rabczynski J, et al. Intragraft mRNA Expression of Cytokines and Growth Factors in Human Kidney Allograft Biopsies by In Situ RT-PCR Analysis. Transplant Proc (2005) 37:767-9. doi: 10.1016/j.transproceed.2005.01.082

40. Pribylova-Hribova P, Kotsch K, Lodererova A, Viklicky O, Vitko S, Volk HD, et al. TGF-Betal mRNA Upregulation Influences Chronic Renal Allograft Dysfunction. Kidney Int (2006) 69:1872-9. doi: 10.1038/sj.ki.5000328

41. Viklicky O, Hribova P, Volk HD, Slatinska J, Petrasek J, Bandur S, et al. Molecular Phenotypes of Acute Rejection Predict Kidney Graft Prognosis. J Am Soc Nephrol (2010) 21:173-80. doi: 10.1681/ASN.2008121268

42. Magil AB. Monocytes/macrophages in Renal Allograft Rejection. Transplant Rev (Orlando) (2009) 23:199-208. doi: 10.1016/j.trre.2009.06.005

43. Papadimitriou JC, Drachenberg CB, Munivenkatappa R, Ramos E, Nogueira J, Sailey C, et al. Glomerular Inflammation in Renal Allografts Biopsies After the First Year: Cell Types and Relationship With Antibody-Mediated Rejection and Graft Outcome. Transplantation (2010) 90:1478-85. doi: 10.1097/ TP.0b013e3181ff87f5

Conflict of Interest: The authors declare that the research was conducted in the absence of any commercial or financial relationships that could be construed as a potential conflict of interest.

Publisher's Note: All claims expressed in this article are solely those of the authors and do not necessarily represent those of their affiliated organizations, or those of the publisher, the editors and the reviewers. Any product that may be evaluated in this article, or claim that may be made by its manufacturer, is not guaranteed or endorsed by the publisher.

Copyright $\odot 2021$ Trailin, Mrazova, Hruba, Voska, Sticova, Slavcev, Novotny, Kocik and Viklicky. This is an open-access article distributed under the terms of the Creative Commons Attribution License (CC BY). The use, distribution or reproduction in other forums is permitted, provided the original author(s) and the copyright owner(s) are credited and that the original publication in this journal is cited, in accordance with accepted academic practice. No use, distribution or reproduction is permitted which does not comply with these terms. 